\title{
LVIII. Meteorlogical register kept at Funchal, in Madeira, in the year 1826; with some prefatory observations on the climate of that Island, \&c
}

\section{Heineken M.D.}

To cite this article: C. Heineken M.D. (1827) LVIII. Meteorlogical register kept at Funchal, in Madeira, in the year 1826; with some prefatory observations on the climate of that Island, \&c , Philosophical Magazine Series 2, 2:11, 362-374, DOI: 10.1080/14786442708674499

To link to this article: http://dx.doi.org/10.1080/14786442708674499

曲 Published online: 10 Jul 2009.

Submit your article to this journal $[\pi$

Џll Article views: 2

Q View related articles $\sqsubset$ 
LVIII. Meteorological Register kept at Funchal, in Madeira, in the Year 1826; with some prefatory Observations on the Climate of that Island, \&c. By C. Heineken, M.D.*

GUNCHAL forms the area to a natural amphitheatre on the south side of the island of Madeira : it is open to the sea towards the south, and shut in on the north by mountains rising to the height of 5000 feet, and gradually declining east and west.

The following Journal is, I am aware, imperfect; but when I plead continued ill-health on the one hand, and renounce all scientific pretensions on the other, I trust that it will be received with every allowance. Mr. Kirwan's observations on the climate of Madeira, I have never seen. Those published by Dr. Gourlay, twenty-five years ago, were made by the late Mr. James Murdoch, at a place called the Valle, about 400 feet above the level of the sea, and do not therefore apply to the city of Funchal : besides, they are given only in monthly maxima, minima, and means; and we are left totally in the dark as to the mode by which these different results were obtained. Mr. Bowdich in his " History of Madeira," did not pretend to enter into this subject, in detail ; and I am not conscious that any other person has attempted it. These, and the intermediate situation of Madeira, which makes it an important connecting link between the tropical and northern climates, are the reasons which have induced me to obtrude myself upon the public, as one who has incurred the sarcasm of the amiable Johnson, by "registering the changes of the wind, in order to die fully convinced that the wind is changeable!"

The periods of the day at which the observations have been made, are not those which I should have selected, had a free choice been offered me; but all others were precluded, either by the state of my health, or the nature of my avocations. I selected therefore the least eligible, because they were likely to prove the least interrupted.- As it regards the barometer, the results have not, I think, been greatly influenced by this circumstance; its oscillations during the four-and-twenty hours being comparatively, and upon an average, so trifling: but the deductions from the hygrometer are I fear of but little value; one observation of this instrument during the day is obviously inadequate, and I have not therefore attempted to form a mean from it. I regret this the more, because it is a very interesting, and here at least nearly novel, subject of investiga-

\footnotetext{
* Communicated by the Author.
} 
tion; and because the instrument challenges and deserves every attention-an observation after sun-set was for at least nine months in twelve incompatible with health, and one at noon or a few hours later would necessarily have been so often interrupted, that it would have been an incumbrance rather than otherwise. During many of our heavy rains it (the hygrometer) has shown several degrees of dryness, but this has invariably occurred with the wind at N. or N.W.; at S. or S.W. it has seldom reached a degree before deposition has taken place; and this apparent discrepancy is, I think, fully accounted for, by supposing that as the former (the N. and N.W. rains) originate upon, and come to us from, the mountains; we are drenched by rains which belong (if I may be allowed the term) to those regions, our own immediate atmosphere remaining for a time at least below the point of saturation. Throughout the year 1826 the sirocco visited us seldom, and then was generally either incomplete in its character or partial in its influence; but in a former year the hygrometer during its prevalence once showed 45 degrees of dryness, and even then æether failed to produce a deposition. I take for granted that it is very well established; although I am ignorant of the explanation, why the sirocco here is so perfectly dry, and that of the Mediterranean so loaded with moisture. It reaches us immediately from the coast of Africa, after passing over about 300 miles of sea: not a cloud is to be seen during its continuance; the whole atmosphere is of one uniform unvaried blue, of a peculiar character, as though viewed through what a painter would term a very thin warm aërial haze; it blows from the E.S.E., lasts almost invariably three days, and encounters you like the puffs from the mouth of an oven or furnace; the eyes and lips feel much as they do when exposed to a keen easterly wind on a frosty day in a northern climate: birds and insects seem to suffer from it more or less, and fowls confined in a close yard generally droop. Furniture warps and cracks; books gape as they do when exposed to a fire, and it is generally inconvenient and oppressive; but I have never heard (although no precautions are taken to avoid it by the labouring classes) that any ill effects have been produced by it. Some have asserted that it has raised the thermometer as high as $130^{\circ}$ in the sun, and $95^{\circ}$ in the shade; but $I$ doubt the accuracy of the observations: for in the course of four years I have never seen it raised above $85^{\circ}$ in perfect shade. There is, however, nothing in which observers are so likely to differ as in their results of the maximum heat in the shade in this place. I do not believe that it is to be accurately obtained, but by a series of observa3 A 2 
tions, made upon several instruments, in varions situations; for the sum is so vertical that no one thermometer permanently fixed in one given place, can remain during the four-and-twenty hours uninfluenced by either its direct or reflected rays:-few persons would, and none I believe hitherto have, attempted such a troublesome mode of accuracy; and as I only profess to five the indications of a single self-regristering instrument, I have, after many trials in a variety of situntions, adopted that which appears to be the least objectionable; namely, an unoccupied room, having another over it (for if only covered by the roof it will be palpably influenced by the sun), and the door and windows of which are constantly open. As to the probable average of rain which one year with another falls at the level of Funchal, I incline to differ from $\mathrm{Mr}$. Bowdich. He estimates it at 10 inches, and it is true that 43.35 inches bave this year fallen; but so much rain has not before been remembered by the oldest inhabitant, and last year the amount was only 20.43 inches, making an average of $31 \cdot 89$. 30 inches I should therefore consider much nearer to the truth. The sutumal rains commence generally towards the end of September, and terminate in December; they have more the character of violent and intermitting showers, than incessant daily raius. The winter rains set in, and prevail more or less throughout January and February, and are far more decided, and tropical. March and April are showery and windy. May tine, with a passing shower ; and in June, July, August, and part of September, we seldom have a drop of rain. At the level of the city no perceptible dew is produced; but up the mountains it is profuse, and all meteorological observations are here circumscribed to a degree which is unknown in an extensive and tolerably plain country. Tables for Funchal belong to that locality only, and cannot in any way be made to suit the island generally; and until a series of observations shall be made at different heights and in different aspects, it is mere deception to apply any deductions to Madeira as a rohole. At the moment that I am writing the sun is shining, and in these lower regions the day is lovely; sea-ward the atmosphere is cloudless, and there is more need of a parasol than an umbrella: but I have only to look out from a north window, to see it raining in torrents upon the summits of the mountains.

For the hourly observations of the barometer on the 4th and 5th of December I am indebted to Mr. Wilkinson, a gentleman who to accuracy and scientific knowledge adds a most enviable share of industry and perseverance. I am fully sensible that standing by theniselves they are of little compar 
rative value, but $I$ hope ere long to obtain others from the same source. The symprisometer I obtained accidentally, and in time only for the December observations: but as it is to me a new instrument, I know nothing of its merits, and give the results merely because I happened to note them at the time as a matter of inquiry. It has hitherto agreed very nearly on an average with what I believe to be an accurate barometer, and differed but slightly (after allowing for corrections) in each observation; and it certainly possesses an advantage in correcting itself for temperature. During three years the highest at which the barometer has ever stood is $30^{\circ} 62 ;-$ in Gourlay's tables $31 \cdot$ is mentioned: but at 400 feet above the sea (the height of Mr. Murdoch's instrument), I will venture to assert that such a phænomenon never occurred even a quarter of a century ago. $28 \frac{1}{2}$ inches are also given by him as the lowest; but when I observe in the same tables $26^{\circ} 9$ (an evident misprint), I cannot but be charitable enough to give him the benefit of a compositor's blunder, especially when during the period before mentioned (three years) 29.39 is the lowest point to which the mercury has ever descended, and when it was then so notoriously low as to be the theme of general remark with several who paid attention to the matter. Generally the barometer is highest with a N.E. wind, and lowest with a S.W.; the great predominance of the first will, perhaps, appear extraordinary; but they are in fact the skirtings of the trade-winds. I do not pretend to great accuracy as to the precise points of the compass from which the winds have blown; upon a mountainous speck in the ocean such as this, the only method of judging is to look to sea with a glass; for all indicators on shore, or in the bay, serve only to deceive. During the greater part of the year we have a sea and land breeze morning and evening; and with this exception it is rare that the wind changes more than once during the fourand-twenty hours; for weeks, sometimes almost for months without intermission, it will come from the N., N.E., and E., and then we have our finest weather; to the S. of $\mathrm{F}$. we look for a sirocco, and if it lingers at the $S$. or passes to the $W$. of it, heavy warm rain invariably follows, and continues as long as it remains in that quarter; to the N. of W. it becomes more violent, and always attended with cold, squally, severe (for this climate) weather; and in the winter with snow upon the summits of the mount:fins, and most of the little thunder and lightning which occur here-reaching the $\mathbf{E}$. of $N$. it becomes again fine; and the sailor's mode of judging of the weather is here peculiarly applicuble. I never remember a 
week's sunshine when the wind had passed from the W. to the N.E. by the S. No snow has yet appeared upon the mountains (December 31 st), but the winter has notwithstanding been severe. More thunder and lightning has occurred than usual, for we have very little; indeed, a gold-leaf electrometer (not however in a favourable situation) has seldom been at all affected, and then only very slightly.

The quantity of rain from the eastward was so unusual a circumstance as to excite the observation and astonishment of every one. As a general law, to which but few exceptions will arise, it may be said that our rains are periodical, and that they oome from the westward of N. and S.; and that to the eastward of those points of the compass it is all fine open weather. I have perhaps been needlessly precise in the description of the instruments used, and their positions; but as I felt that my name could give no weight to their results, I was unwilling to deprive them of the benefit which could accrue from a certificate of the mode in which they were conducted.

In the course of the summer I ascended Pico Ruivo, the highest point of the island, and by Newman's iron cistern bam rometer and Daniell's hygrometer made the height 6069 feet above the level of the sea; See Note $(a)$. Bowdich gives it as 6164 feet; but then he states Mr. Veitch's turret; where his lower instrument hung, to be 154 feet in height, whereas il appears to me to be only 97 ; See Note (b). Admitting this to be the case we agree within 38 feet; no great matter of difference in such a height and under such opposite circumstances; for our instruments were different, and our data taken from contrary sides of the island: it should in candour also be stated; that my observations were conducted with one barometer only; and should his height of the turret appear to have been erroneous, the error I am presuaded did not originate with him; for when already embarked for Africa, he wrote me a note which has been destroyed, and the particulars of which I cannot now remember, requesting me to desire his publisher to make some alteration in the height of that identical turret. What this alteration was I cannot recall to mind; but I have no doubt that the publisher neglected to make the correction, and that the error is thus satisfactorily accounted for.

Funchal, Madeira, Dec. 31, 1826 . C. Heineken, M.D. 


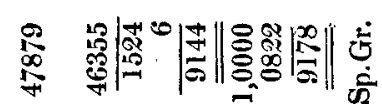

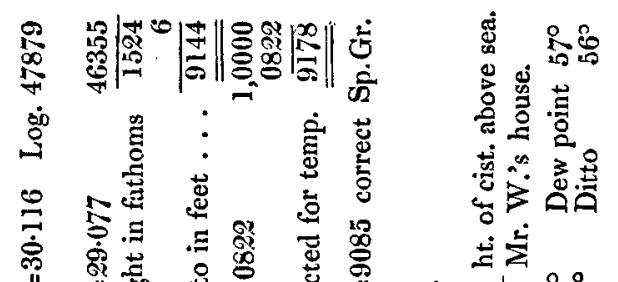

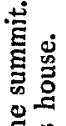

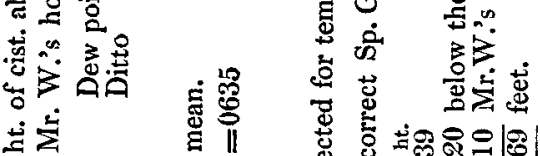

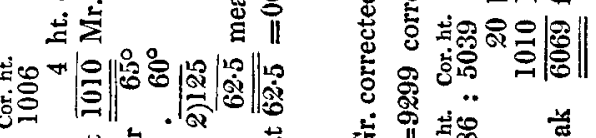

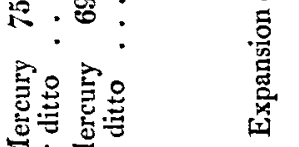

का

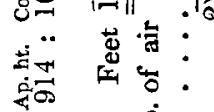

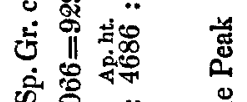

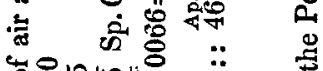

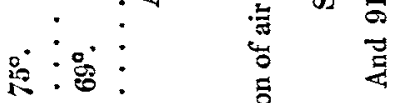

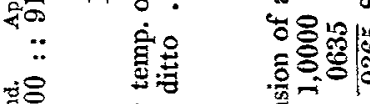

i:

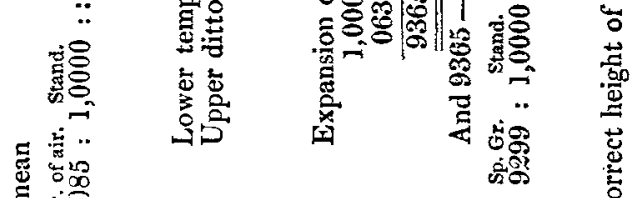

는

웜

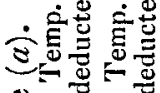

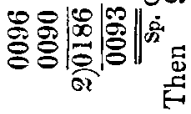

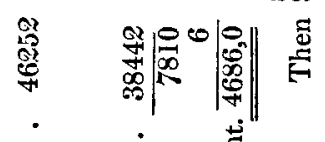

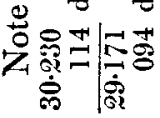

离: $\vdots$ : 只品

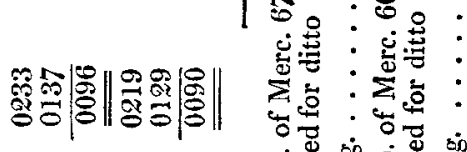

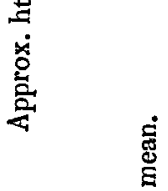

?

$\vdots \vdots$ 言。

$\vdots \vdots \vdots$ 等

$\ldots$

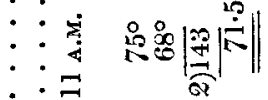

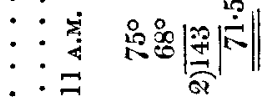

亲:

政

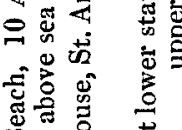

๓ं के

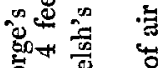

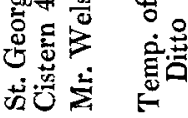

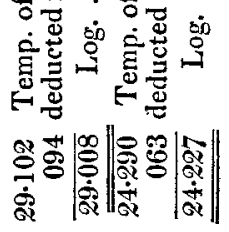

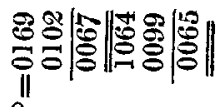

웅

政

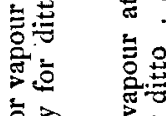

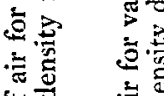

क्षे

-

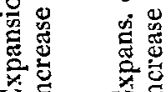

宛

迹

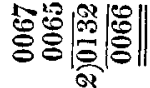

范 


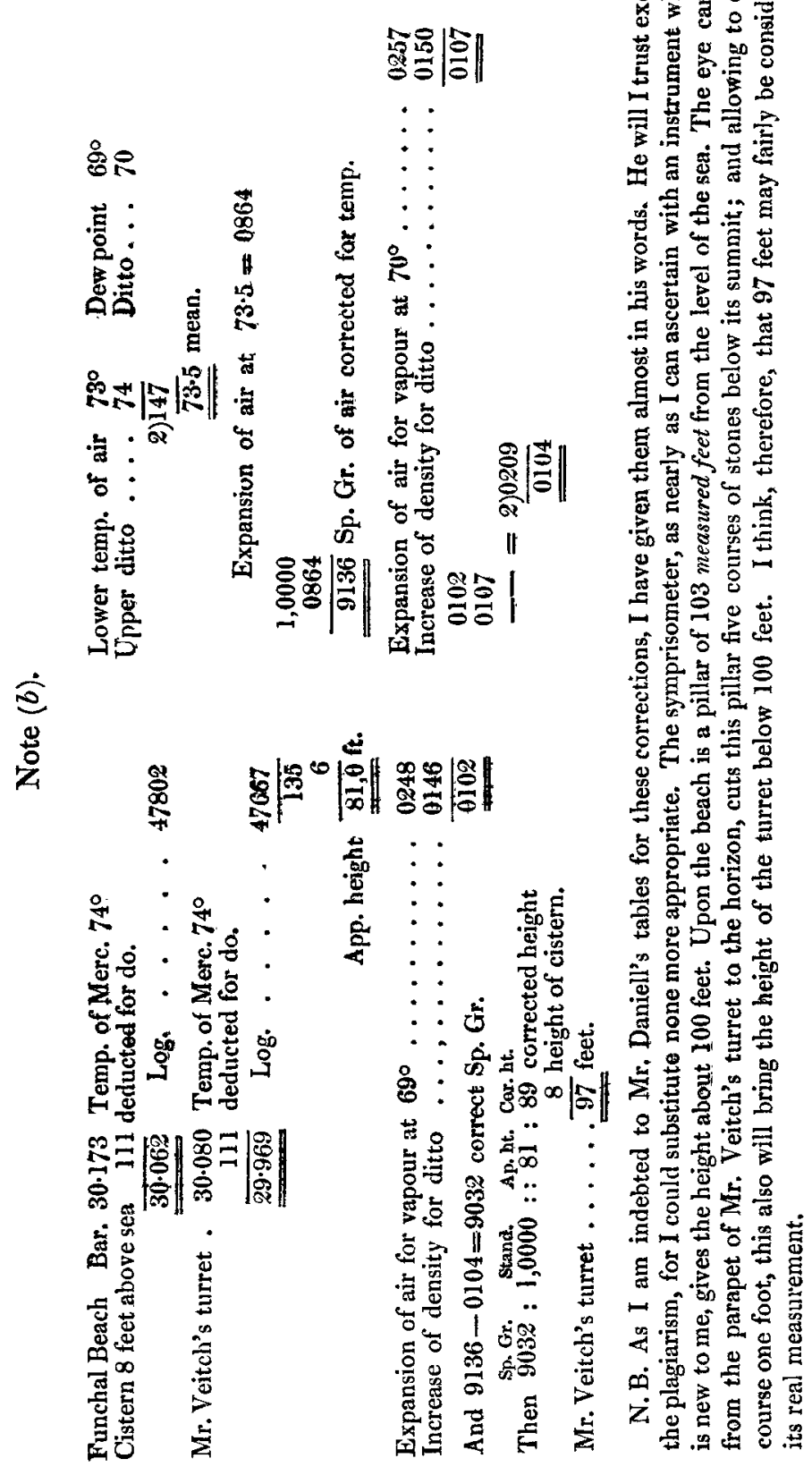


J A N U A R Y 1896.

\begin{tabular}{|c|c|c|c|c|c|c|c|c|c|c|}
\hline \multicolumn{5}{|c|}{10 o'Clock A.M. } & \multicolumn{3}{|c|}{10 o'Clock e.M. } & \multirow{3}{*}{ Rain. } & \multirow{3}{*}{ Wind } & \multirow{3}{*}{ Weather. } \\
\hline \multicolumn{2}{|c|}{ Barometer. } & \multicolumn{3}{|c|}{ Hygrometer } & \multicolumn{2}{|c|}{ Barometer. } & \multirow{2}{*}{$\frac{\text { Ther. }}{+-1}$} & & & \\
\hline Bar. & Th. & Aur & $\underline{D}$ & ID & Bar. & Th. & & & & \\
\hline 130.23 & $\overline{62}$ & & & & $30 \cdot 15$ & 63 & 6453 & & w. & Fine \\
\hline $230 \cdot 20$ & 62 & & & & $30 \cdot 13$ & 63 & 6455 & & NW. & Show \\
\hline $3 \mid 30 \cdot 02$ & 63 & & & & $29 \cdot 80$ & 65 & 6758 & & w. & Showers [on $\mathrm{m}^{\mathrm{ns}}$ \\
\hline $4 \cdot 29 \cdot 70 \mid$ & 63 & & & & $29 \cdot 63$ & 62 & 6650 & $1 \cdot 30$ & Nw. & $\mathrm{H}^{\mathrm{y}} \mathbf{r}^{\mathrm{n}}$ with snow \\
\hline $5|29 \cdot 70|$ & 61 & & & & $29 \cdot 80$ & 61 & 6752 & & Nw. & Showers \\
\hline $629 \cdot 87$ & 60 & & & & $29 \cdot 79$ & 60 & 6353 & .41 & NW. & Rain \\
\hline $7|29 \cdot 83|$ & 60 & & & & $29 \cdot 90$ & 61 & $65 \mid 53$ & & N. & Cly snow $n^{y}$ \\
\hline $830 \cdot 10$ & 60 & & & & $30 \cdot 10$ & 61 & 6952 & & N. & Fine \\
\hline $9 \cdot 30 \cdot 10$ & 60 & & & & $30 \cdot 04$ & 61 & 6755 & & w. & Small rain \\
\hline $1030 \cdot 11$ & 61 & & & & $30 \cdot 13$ & 64 & 6654 & .06 & w. & Fine \\
\hline $1130 \cdot 10$ & 62 & & & & $30 \cdot 05$ & 64 & 0757 & & w. & Slight showers \\
\hline $1230 \cdot 12$ & 65 & & & & $30 \cdot 12$ & 65 & 6754 & $\cdot 05$ & w. & Slight showers \\
\hline $1330 \cdot 14$ & 63 & & & & $30 \cdot 18$ & 62 & $67|55|$ & & w. & Overcast \\
\hline $14 \mid 30 \cdot 23$ & 62 & & & & $30 \cdot 25$ & 64 & 6752 & & NE. & Eind \\
\hline $1530 \cdot 24$ & 62 & & & & $30 \cdot 23$ & 63 & 6857 & & E. & Fine \\
\hline $16 \mid 30 \cdot 20$ & 62 & & & & & & 66.57 & & SE. & Fine \\
\hline $1730^{\circ} 00$ & 63 & & & & 29.85 & 63 & $65 \mid 58$ & $\cdot 10$ & SE. & Overc.P.M.thun ${ }^{r}$ \\
\hline $18 \mid 29 \cdot 85$ & 63 & & & & $29 \cdot 94$ & 63 & 6458 & $\cdot 17$ & SE. & Showers \\
\hline $1930 \cdot 00$ & 63 & & & & $30 \cdot 11$ & 63 & 6760 & & SF.. & Overcast \\
\hline $20 \mid 30 \cdot 15$ & 62 & 62 & 50 & 12 & $30 \cdot 10$ & 62 & 6857 & & SE. & Orercast \\
\hline 2130.08 & 63 & 62 & 53 & 9 & $30 \cdot 06$ & 62 & 6455 & & E. & Overcast \\
\hline $22|30 \cdot 07|$ & 61 & 62 & 52 & 10 & $50 \cdot 12$ & 62 & 66.53 & & E. & Overcast \\
\hline $330 \cdot 20$ & 61 & 61 & 52 & 9 & $30 \cdot 20)$ & 62 & 6752 & & NE. & Fine \\
\hline $2430 \cdot 23$ & 61 & 61 & 46 & 15 & $30 \cdot 21$ & 61 & 6652 & & NE. & Fine \\
\hline $25 \mid 30 \cdot 19$ & 61 & 61 & 43 & 18 & $30 \cdot 03$ & 62 & 64.51 & & w. & Do. P.M. shower \\
\hline $26-29 \cdot 88$ & 61 & 60 & 53 & 7 & $29 \cdot 91$ & 60 & 63.54 & .34 & $N w$. & Showers, snow \\
\hline $2729 \cdot 98$ & 61 & 61 & 51 & 10 & $30 \cdot 01$ & 60 & 6652 & & NW. & Fine [oñmonnts \\
\hline $28 \mid 30 \cdot 11$ & 61 & 60 & 46 & 14 & $30 \cdot 09$ & 61 & 6453 & & NW. & Fine \\
\hline $29 \mid 30 \cdot 07$ & 60 & 60 & 55 & 5 & $30 \cdot 00^{\circ}$ & 61 & 6053 & $\cdot 63$ & Nw. & Do. P.M. rain \\
\hline $30|30 \cdot 15|$ & 60 & 60 & 52 & 8 & $30 \cdot 20 \mid$ & 60 & 6653 & & Nw. & Sh ${ }^{r}$ much snow \\
\hline $31,29 \cdot 94$ & 59 & 60 & 57 & 3 & & & 6453 & 220 & w. & Heavy rain \\
\hline
\end{tabular}

Pressure. Corrd for Expans.

Max. $30 \cdot 25$ at $64^{\circ}=30 \cdot 165$

Min. $29 \cdot 630 \quad 62=29.547$

Mean $30 \cdot 049 \quad 62=29 \cdot 977$

Temperature.

Max. ......................6 $69^{\circ}$

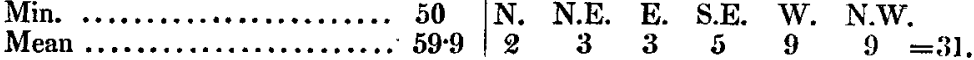

Remarks.-A severe winter month, with much more snow than usual; on 31 st the Hygrometer showed $3^{\circ}$ of dryness during the heaviest and most universal rain that has occurred for some time.

New Series. Vol. 2. No. 11. Nov. 1827.

Dew Point.

Max. $57^{\circ}$ Min. $43^{\circ}$

Max. $18^{\text {Dryness. }}$ Min. 3

Rain 5.32 inches.

Winds. 
F E B R U A R Y 1826.

\begin{tabular}{|c|c|c|c|c|c|c|c|c|}
\hline \multicolumn{3}{|c|}{10 o'Clock A.M. } & \multicolumn{3}{|c|}{10 n'Clock P.M. } & \multirow{3}{*}{ Rain. } & \multirow{3}{*}{ Wind } & \multirow{3}{*}{ Weather. } \\
\hline \multirow{2}{*}{\begin{tabular}{|l|} 
Barometer. \\
Bar. |'Th.
\end{tabular}} & \multicolumn{2}{|c|}{ Hygrometer } & Barometer. & \multicolumn{2}{|c|}{ Therm. } & & & \\
\hline & \begin{tabular}{|l|l} 
Air. & D.P.P. \\
\end{tabular} & Dry. & $\begin{array}{ll}\text { Bar. } & \text { Th. } \\
\end{array}$ & & $E$ & & & \\
\hline \begin{tabular}{l|l|l|l|}
1 & $29 \cdot 9$ & 59
\end{tabular} & 6051 & 9 & 30.0360 & 62 & 53 & & w. & Fine \\
\hline $2|30 \cdot 16| 60$ & $60 \quad 55$ & 5 & $\mid 30 \cdot 2361$ & 62 & 54 & & w. & Fine \\
\hline $3 \mid 30 \cdot 2461$ & \begin{tabular}{|l|l|}
61 & 57
\end{tabular} & 4 & 30.2363 & 63 & $53 \mid$ & & w. & Overcast \\
\hline $4|30 \cdot 37| 61$ & $61 \mid 40$ & 21 & $\mid 30 \cdot 41162$ & 63 & 53 & & E. & Fine \\
\hline $5|30 \cdot 46| 62$ & $62 \mid 50$ & 12 & 30.4662 & 63 & 54 & & NE. & Fine \\
\hline $6|30 \cdot 476| 62$ & 6251 & 11 & $30 \cdot 4562$ & 64 & 54 & & NE. & Fine \\
\hline $7|30 \cdot 42| 62$ & \begin{tabular}{|l|l|}
62 & 54
\end{tabular} & 8 & 30.3963 & 63 & 58 & & NE. & Fine \\
\hline $8|30 \cdot 4162 \cdot 5|$ & $63 \mid 59$ & 4 & $30 \cdot 3 \bar{v} 62 \cdot 5$ & 64 & 56 & & w. & Overcast \\
\hline $9|30 \cdot 26| 62 \cdot 5 \mid$ & $63 \mid 58$ & 5 & $|30.21| 63$ & 62 & $51\}$ & & w. & Fine \\
\hline $10|30 \cdot 16| 62$ & \begin{tabular}{l|l|}
62 & 48
\end{tabular} & 14 & $30 \cdot 1561$ & 62 & 52 & & NW. & Do. in town, \\
\hline $11|30 \cdot 23| 61 \cdot 5 \mid$ & 6254 & 8 & $30 \cdot 3161$ & 63 & 55 & & Nw. & Do. [sn. hills \\
\hline $12|30 \cdot 40| 62$ & \begin{tabular}{|l|l|}
62 & 59
\end{tabular} & 3 & $|30.40| 63$ & 63 & 51 & & Nw. & \\
\hline $3|30 \cdot 4.2| 61 \cdot 5 \mid$ & $62 \mid 54$ & 8 & $|30.38| 62$ & 63 & $55 \mid$ & & E. & Fine \\
\hline $4|30 \cdot 35| 61 \cdot 5$ & $62 \lcm{55}$ & 7 & $|30.31| 62$ & 63 & 56 & & w. & Overcast \\
\hline$|15| 30 \cdot 35 \mid 62$ & $62 \mid 57$ & 5 & $\mid 30.4163$ & 63 & 55 & & NW. & Fine \\
\hline $16|30 \cdot 50| 63$ & 6455 & 9 & $30.50 \mid 64$ & 65 & 55 & & E. & Fine \\
\hline 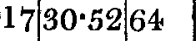 & \begin{tabular}{l|l|}
655 & 56
\end{tabular} & 9 & $|30.55| 67$ & 65 & $56 \mid$ & & E. & Fine \\
\hline $18|30.59| 64$ & $65 \mid 52$ & 13 & 30.5964 & 64 & 53 & & E. & Fine \\
\hline$|19| 30 \cdot 56 \mid 63$ & $65 \mid 52$ & $12 \mid$ & $30 \cdot 50,64$ & 64 & 52 & & E. & Fine \\
\hline \begin{tabular}{|l|l|l|}
20 & $30 \cdot 4 \cdot 7$ & 63
\end{tabular} & $\begin{array}{l:l}64 & 50 \\
\end{array}$ & 14 & $30 \cdot 4863$ & 64 & $|51|$ & & E. & Fine \\
\hline \begin{tabular}{|l|l|l|l}
21 & 30.51 & 63
\end{tabular} & $64 \mid 58$ & 5. & $30 \cdot 51 \mid 63$ & $63 \cdot 5$ & $52 \mid$ & & E. & Fine \\
\hline $22|30 \cdot 52| 63$ & $63 \mid 58$ & 5 & $30 \cdot 4963$ & 64 & 53 & & NE. & Fine \\
\hline $23|30 \cdot 46| 63$ & 6354 & 9 & $\mid 30 \cdot 4,462$ & 63 & $|56|$ & & E. & Overcast \\
\hline $24 \mid 30 \cdot 4963$ & 64.52 & 12 & $30 \cdot 4664$ & 64 & 53 & & E. & Fine \\
\hline $25|30 \cdot 45| 63 \cdot 5 \mid$ & $64 \mid 51$ & 13 & $30 \cdot 4064$ & 65 & 53 & & NE. & Fine \\
\hline $26|30: 31| 63 \cdot 5 \mid$ & $64 \cdot 51$ & 13 & 30.2564 & 64 & 53 & & E. & Fine \\
\hline $27|30 \cdot 29| 63 \cdot 5 \mid$ & $64: 52$ & 12 & 30.2964 & 66 & 55 & & E. & Fine \\
\hline $28|30 \cdot 35| 65$ & 68 & 24. & $|30 \cdot 30| 66$ & 68 & 63 & & SE. & Sirocco \\
\hline
\end{tabular}

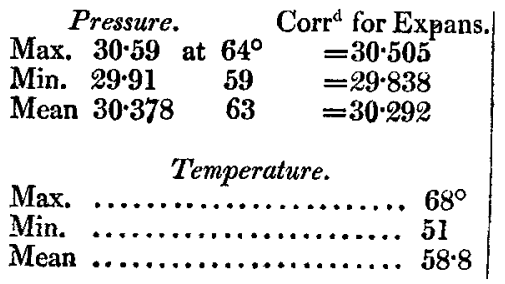

Max. $59^{\circ}$ ew Point.

Max. $24^{\text {Dryness. }}$ Min. 3

Rain none.

Winds.

N.E. E. S.E. W. N.W.

$5 \quad 12 \quad$ I $6 \quad 4=28$.

Remarks. - A very fine open month. 
M A R C H 1826.

\begin{tabular}{|c|c|c|c|c|c|c|c|}
\hline \multicolumn{3}{|c|}{10 o'Clock A.M. } & \multicolumn{2}{|c|}{ J0 o'Clock P.м. } & \multirow{3}{*}{ Rain. } & \multirow{3}{*}{ Wind } & \multirow{3}{*}{ Weather. } \\
\hline Barometer. & \multicolumn{2}{|c|}{ Hygrometer } & Barometer. & \multirow{2}{*}{ Ther. } & & & \\
\hline Bar. Th. & Air. D.P & Dry. & Bar. Th. & & & & \\
\hline $130 \cdot 2565$ & 7040 & 30 & $|30 \cdot 22| 66$ & $68 \mid 58$ & & SE. & Siro \\
\hline $0 \cdot 23 \mid 66$ & $67 \mid 55$ & 12 & $|30 \cdot 20| 66$ & 6755 & & SE. & Very \\
\hline 330.2365 & \begin{tabular}{|l|l}
65 & 57
\end{tabular} & 8 & $|30.23| 66$ & $67 \mid 56$ & & NE. & Fine \\
\hline $4 \mid 30 \cdot 2565$ & 66.53 & 13 & $\mid 30.2766$ & 6757 & & NE. & Fine \\
\hline $0 \cdot 34 \mid 66$ & $66 \mid 60$ & 6 & $|30 \cdot 32| 67$ & 6758 & & E. & Fine \\
\hline \begin{tabular}{l|l|l|}
6 & $30 \cdot 30$ & 66
\end{tabular} & 67.50 & 17 & $|30 \cdot 21| 66$ & 6856 & & NE. & Fine \\
\hline $7|30 \cdot 15| 65 \cdot$ & $5|67| 49$ & 18 & $30 \cdot 06 \mid 68$ & $68 \mid 60$ & & E. & Fine \\
\hline $8|30.02| 67$ & $67 \mid 57$ & 10 & $|30 \cdot 02| 69$ & 6960 & & E. & Very thick and \\
\hline 0068 & $67 \mid 60$ & 7 & $30 \cdot 05 \mid 69$ & $68 \mid 62$ & & E. & $N$. clear \\
\hline$\cdot 0869$ & \begin{tabular}{|l|l|}
68 & 59
\end{tabular} & 9 & $|30 \cdot 20| 67$ & $68 \mid 56$ & $\cdot 26$ & w. & Show \\
\hline $18 \mid 67$ & \begin{tabular}{|l|l|}
68 & 53
\end{tabular} & 15 & $|30 \cdot 11| 67$ & $67 \mid 56$ & & E. & Fin \\
\hline 567 & 6857 & 11 & 30.0269 & 6957 & & E. & Fine \\
\hline 68 & \begin{tabular}{|l|l|}
68 & 59
\end{tabular} & 9 & $\left|30^{\circ} 04\right| 69$ & $67 \mid 58$ & & E. & $\mathrm{Fi}$ \\
\hline 1067 & 67.56 & 11 & $|30 \cdot 12| 69$ & 6760 & & E. & Fin \\
\hline 1967 & $67 \mid 56$ & 11 & $|30 \cdot 23| 67$ & $67 \mid 58$ & & NE. & Fil \\
\hline 66 & $67 \mid 50$ & 17 & $|30 \cdot 15| 67$ & $67 \mid 57$ & & NE. & Fine \\
\hline 966 & \begin{tabular}{|l|l|}
66 & 49
\end{tabular} & 17 & $30 \cdot 0366$ & $67 \mid 57$ & & w. & hit show \\
\hline .0265 & $65 \mid 55$ & 10 & $30 \cdot 0365$ & $67[57$ & $\cdot 10$ & Nw. & Shor \\
\hline 1066 & $66 \mid 55$ & 11 & $|30 \cdot 12| 65$ & $67 \mid 57$ & 0.5 & NW. & Showe \\
\hline 65 & $65 \mid 56$ & 9 & $|30 \cdot 11| 68$ & $67 \mid 55$ & & NW. & e \\
\hline 65 & $65 \mid 53$ & 12 & $|30 \cdot 13| 65$ & 6654 & & w. & Fin \\
\hline 1964 & \begin{tabular}{|l|l|}
65 & 4.7
\end{tabular} & 18 & $\left|30^{\circ} 16\right| 65^{\circ}$ & $566 \mid 56$ & & Nw. & Fir \\
\hline \begin{tabular}{|l|l|l|l|}
23 & $30 \cdot 10$ & 64
\end{tabular} & 64.4 & 19 & $29 \cdot 90 \mid 67$ & $67 \mid 53$ & $\cdot 42$ & Nw. & Do., \\
\hline 7864 & 6458 & 6 & $|29 \cdot 68| 66$ & 64.52 & •66 & w. & iin, sn. \\
\hline 7963 & $64 \mid 52$ & 12 & $|29 \cdot 85| 65$ & $64 \mid 52$ & $0 \bullet 6$ & w. & Fine \\
\hline 9461. & $61 \mid 47$ & 14 & $30 \cdot 0161$ & 6451 & & NW. & Fine \\
\hline $.07 \mid 61$ & \begin{tabular}{|l|l|}
61 & 4.6
\end{tabular} & 15 & $|30.07| 61.5$ & $563 \mid 53$ & • 40 & Nw. & Rain at night \\
\hline \begin{tabular}{|l|l|l|}
28 & $29 \cdot 93$ & 62
\end{tabular} & \begin{tabular}{|l|l|}
62 & 60
\end{tabular} & 2 & $29 \cdot 81 \mid 62$ & 6355 & $1 \cdot 73 \mid$ & w. & $\mathrm{Ra}$ \\
\hline 7963 & $64 \mid 60$ & 4 & $|29 \cdot 92| 62$ & 64.55 & $0 \cdot 4$ & SE. & Shower $\left[\right.$ show $^{\mathrm{r}}$. \\
\hline & \begin{tabular}{|l|l|}
62 & 50 \\
\end{tabular} & 12 & $30 \cdot 05 \mid 61$ & $63 \mid 55$ & & E. & Fine, $P$. \\
\hline$|31| 30 \cdot 03 \mid 62$ & $|63| 49 \mid$ & 14 & $|30 \cdot 11| 66$ & $65 \mid 54$ & & NE. & PO \\
\hline
\end{tabular}

Pressure.

Max. 30.34 at $66^{\circ}=\mathbf{3 0} \cdot 254$

Min. $29 \cdot 68 \quad 66=29 \cdot 595$

Mean $30.083 \quad 66=29 \cdot 998$

Temperature.

Max. ....................6 $69^{\circ}$

Min. .................. 51

Mean $. . . \ldots \ldots \ldots \ldots \ldots \ldots \ldots \ldots, 61 * 3$
Dew Point.

Max. $60^{\circ} \quad$ Min. $40^{\circ}$

Max. $30^{\text {Dryness. }}$ Min. 2

Rain $3 \cdot 72$ inches.

Winds.

N.E. E. S.E. W. N.W.

$\begin{array}{lllll}6 & 9 & 3 & 5 & 8\end{array}=31$.

Remarks. - The former part of the month was warmer and more settled than has been long remembered; from the 16 th it was as usual, rainy and windy. 
A P R I L 1826.

\begin{tabular}{|c|c|c|c|c|c|c|c|}
\hline \multicolumn{3}{|c|}{10 o'Clock A.m. } & \multicolumn{2}{|c|}{10 o'Clock P.M. } & \multirow{3}{*}{ Rain. } & \multirow{3}{*}{ Wind } & \multirow{3}{*}{ Weather. } \\
\hline Baroineter. & \multicolumn{2}{|c|}{ Hygronieter } & Barometer. & Ther. & & & \\
\hline 1 Bar. Th. & Air. D.P.I & Dry. & Bar. & Th. $\pm=$ & & & \\
\hline $130 \cdot 0262 \cdot 5$ & 6455 & 9 & $30 \cdot 06$ & 656456 & & NE. & Fine \\
\hline $230 \cdot 0964$ & $65|57|$ & 8 & $30 \cdot 15$ & $6764 \mid 56$ & & NE. & Fine \\
\hline $330 \cdot 1864 \cdot 5$ & $67|52| 1$ & 15 & $30 \cdot 21$ & $65|6555|$ & & E. & Fine \\
\hline $430 \cdot 2163$ & $65 \mid 56$ & 9 & $30 \cdot 18$ & $65 \quad 6555$ & & w. & Fine \\
\hline $530 \cdot 1865$ & \begin{tabular}{ll|l}
67 & 49 & 1
\end{tabular} & 18 & $30 \cdot 16$ & 676660 & & SE. & Partial sirocco \\
\hline $630 \cdot 10 \mid 66$ & 6947 & 22 & $30 \cdot 10$ & $66|67| 65 \mid$ & & SE. & Partial sirocco \\
\hline $7\left|30^{\circ} 07\right| 66$ & \begin{tabular}{|l|l|}
67 & 55
\end{tabular} & 12 & $30 \cdot 12$ & 656657 & & E. & Fine \\
\hline $830 \cdot 1465$ & $67|50| 1$ & 17 & $30 \cdot 17$ & $6566|57|$ & & NE. & Fine \\
\hline 9$] 30 \cdot 20 \mid 65$ & 6650 & 16 & $30 \cdot 26 \mid$ & 656657 & & NE. & Fine \\
\hline $10|30.22| 65$ & $66|53| 1$ & 1.3 & $30 \cdot 21$ & 676658 & & NE. & Fine \\
\hline $11|30 \cdot 21| 65 \cdot 5 \mid$ & $65 \quad 59$ & 6 & $\mid 30 \cdot 25]$ & $66|67,59|$ & & NE. & Fine \\
\hline \begin{tabular}{l|l|l|l|}
12 & $30 \cdot 29$ & 66
\end{tabular} & $67 \mid 62$ & 5 & $30 \cdot 30$ & $67 \quad 6857$ & & NE. & Fine \\
\hline $13 \mid 30 \cdot 2966$ & $6762 \mid$ & 5 & $30 \cdot 22$ & $68 \quad 68 \mid 62$ & & NE. & Fine \\
\hline $14 \mid 30 \cdot 1467$ & 66,60 & 6 & $30 \cdot 05$ & 676861 & & NE. & Fine \\
\hline $1530 \cdot 0568$ & $\left.|73| 46\right|^{c}$ & 27 & $30 \cdot 06$ & 686864 & & SE. & Siroces \\
\hline $1630 \cdot 1068$ & $7346^{c}$ & 27 & $|30 \cdot 13|$ & $7071 \mid 63$ & & SE. & Siroces \\
\hline $1730 \cdot 0870$ & $72 \mid 52$ & 20 & $30 \cdot 11$ & $71 / 72 \mid 59$ & & w. & Fine \\
\hline $18\left|30^{\circ} 06\right| 69$ & 6862 & 6 & $30 \cdot 01$ & $70|7262|$ & & w. & Fine \\
\hline $19|29 \cdot 97| 69$ & $68|58| 1$ & 10 & $29 \cdot 93$ & $6870|57|$ & & w. & Gine \\
\hline $20,29 \cdot 86 \mid 68$ & $68 \mid 56$ & 12 & $29 \cdot 83$ & 69,7058 & & w. & Fine \\
\hline 2129.8867 & 6753 & 14 & $29 \cdot 99$ & $6768 \mid 55$ & .04 & N. & Wine \\
\hline $2230 \cdot 0865$ & $65 \mid 48$ & 17 & $30 \cdot 12 \mid$ & 676856 & & N. & Fine \\
\hline $2330 \cdot 1065$ & 6450 & 14 & $|30 \cdot 14|$ & $67|66| 57 \mid$ & & N. & Cloudy \\
\hline $2430 \cdot 1365$ & 6548 & 17 & $30 \cdot 19$ & $65 \mid 6657$ & & NE. & Fine \\
\hline $25 \mid 30 \cdot 1965$ & 65.54. & 11 & $30 \cdot 20$ & \begin{tabular}{ll|l|l|}
65 & 66 & 59 \\
\end{tabular} & & NE. & Fine \\
\hline $26|30 \cdot 18| 65$ & 6553 & 12 & $30 \cdot 18$ & 666658 & & NE. & Fine \\
\hline \begin{tabular}{|l|l|l|}
$270 \cdot 17$ & 65
\end{tabular} & 6560 & 5 & $30 \cdot 18$ & $67|67| 58 \mid$ & & NE. & Cloudy \\
\hline $2830 \cdot 1766$ & $66 \quad 59$ & 7 & $30 \cdot 19$ & $6766 \mid 57$ & & N. & Cloudy \\
\hline $2930 \cdot 1866$ & 65.59 & 6 & $30 \cdot 16$ & $67 \quad 69 \mid 59$ & & NE. & Cloudy \\
\hline$|30| 30 \cdot 12 \mid 65$ & $|65| 51 \mid$ & 14 & $|30 \cdot 12|$ & $65 \mid 6660$ & & E. & Cloudy \\
\hline
\end{tabular}

Pressure. Corra for Expans.

Max. $30 \cdot 30$ at $67^{\circ}=30 \cdot 214$

Min. 29.83 $69=29 \cdot 732$

Mean $30 \cdot 140 \quad 67 \quad=29 \cdot 959$

Temperature.

Max. .................. $79^{\circ}$

Vin. $\ldots \ldots \ldots \ldots \ldots \ldots \ldots \ldots \ldots, 55$

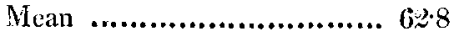

Dew Point.

Max. 620 Min. $46^{\circ}$

Max. $27 \stackrel{\text { Dryness. }}{\text { Min. } 5}$

Rain 0.04 inches.

Winds.

N. N.E. E. S.E. W.

$\begin{array}{lllll}5 & 14 & 3 & 4 & 4=30 .\end{array}$

Remarks.-A fine spring month; the former part remarkably warm. 
M A Y 1826.

\begin{tabular}{|c|c|c|c|c|c|c|c|}
\hline \multicolumn{3}{|c|}{10 o'Clock A.s. } & \multicolumn{2}{|r|}{10 o'Clock P.M. } & \multirow{3}{*}{ Rain. } & \multirow{3}{*}{ Wind } & \multirow{3}{*}{ Weather. } \\
\hline \multirow{2}{*}{$\begin{array}{c}\text { Barometer. } \\
\text { Bar. Th. }\end{array}$} & \multicolumn{3}{|c|}{ Hygrometer. } & Barometer. Ther. & & & \\
\hline & Air. & D.P. I & & Bar. Th. $+1=$ & & & \\
\hline $130 \cdot 0965$ & 65 & $\overline{57} \mid 1$ & & \begin{tabular}{|l|l|l|l|l|}
30.05 & 66 & 66 & 60
\end{tabular} & & E. & Cloudy \\
\hline I 65 & 65 & 48 & 78 & \begin{tabular}{l|l|l|l|}
29.98 & 65 & 66 & 60 \\
\end{tabular} & & NE. & \\
\hline $6 \mid 65$ & 65 & 62 & 3 & \begin{tabular}{|l|l|l|l|}
$29 \cdot 45$ & 66 & 66 & 57
\end{tabular} & .21 & w. & $\mathbf{R}$ \\
\hline $429 \cdot 9665$ & 65 & 60 & 5 & $29 \cdot 97 \cdot 66|6761|$ & & w. & Fine \\
\hline $5 \mid 29 \cdot 9] \mid 66$ & 67 & 59 & 8 & \begin{tabular}{|l|l|l|l|l|}
$29 \cdot \mathrm{s} 6$ & 65 & 66 & 60
\end{tabular} & $\cdot 30$ & sw. & Do.Cly.1 \\
\hline 865 & 65 & 64 & 1 & \begin{tabular}{|l|l|l|l|l|l|l|l}
$29 \cdot 74$ & 66 & 66 & $\mathbf{1}$
\end{tabular} & $1 \cdot 15$ & s. & $\mathbf{R}$ \\
\hline $7|29 \cdot 75| 67$ & 67 & 65 & 2 & \begin{tabular}{|l|l|l|l|l|}
$29 \cdot 84$ & 68 & 68 & 62
\end{tabular} & $\cdot 10$ & $\mathrm{w}$. & Sho \\
\hline $8|29 \cdot 86| 68$ & $67 \cdot 5$ & $656^{2}$ & $2 \cdot 5$ & \begin{tabular}{l|l|l|l|}
$99 \cdot 88$ & 67 & 68 & 60 \\
\end{tabular} & 69 & w. & Rain \\
\hline $929 \cdot 9067$ & 67 & 64 & 3 & $29 \cdot 9467 \mid 6862$ & .25 & NW. & She \\
\hline $1029 \cdot 9367$ & 67 & 65 & 2 & 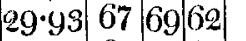 & $\cdot 10$ & w. & \\
\hline i? & 67 & 65 & 2 & 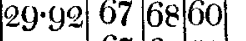 & $\cdot 14$ & w. & \\
\hline $9 \cdot 9167$ & 67 & 61 & 6 & \begin{tabular}{|l|l|l|l|}
$30 \cdot 04$ & 67 & 68 & 55
\end{tabular} & $\cdot 13$ & NE. & Fine \\
\hline $13\left|30^{\circ} 06\right| 65.5$ & 65 & 57 & 14 & \begin{tabular}{|c|c|c|c|}
$30 \cdot 14$ & 65 & 66 & 59 \\
\end{tabular} & & NE. & $\mathrm{Fi}$ \\
\hline $16 \mid 65$ & 66 & 55 & 11 & $|30.20| 65|66| 57 \mid$ & & NE. & dy \\
\hline $20 \mid 65$ & 65 & 56 & 9 & {$\left[\begin{array}{lll|l|l|}30 \cdot 21 & 66 & 67 & 57\end{array}\right.$} & & NE. & $\mathrm{Fi}$ \\
\hline $0 \cdot 23 \mid 65$ & 65 & 521 & 13 & $|30 \cdot 24| 65|66| 57 \mid$ & & NE. & Fir \\
\hline $17|30 \cdot 23| 64 \cdot 5$ & 64 & 54 & 10 & \begin{tabular}{|c|c|c|c|c|}
$30 \cdot 24$ & 65 & 66 & 57
\end{tabular} & & NE. & Fine \\
\hline 364 & 565 & 52 & 13 & $|30 \cdot 23| 65|68| 59 \mid$ & & NE. & $\mathrm{Fi}$ \\
\hline $0 \cdot 23 \mid 66$ & 66 & 56 & 10 & \begin{tabular}{|c|c|c|}
$30 \cdot 21$ & 66 & 66 \\
\end{tabular} & & NE. & $\mathrm{Fi}$ \\
\hline $0 \cdot 18 \mid 66$ & 66 & 62 & 4 & $|30 \cdot 18| 68|68| 58 \mid$ & & NE. & $\mathrm{Cl}$ \\
\hline $0 \cdot 1467$ & 67 & 56 & 11 & \begin{tabular}{|c|c|c|c|}
$30 \cdot 13$ & 67 & 68 & 61
\end{tabular} \mid & & NE. & $d y$ \\
\hline 0967 & 67 & $60 \mid$ & 7 & $30.08 \quad 67 \quad 6$ & & NE. & $F$ \\
\hline 0766 & & & & $|30 \cdot 10| 67 \mid \mathrm{C}$ & & & \\
\hline$\cdot 14 \mid 66$ & & & & $|30 \cdot 15| 67|68| 58 \mid$ & & NE. & $\mathrm{Fi}$ \\
\hline 1566 & & & & \begin{tabular}{|l|l|l|}
$30 \cdot 18$ & 67 & 6
\end{tabular} & & NE. & $\mathrm{Fi}$ \\
\hline & & & & $|30 \cdot 22| 66$ & & N. & \\
\hline 265 & & & & $|30 \cdot 19| 66 \quad 68|59|$ & & NE. & $\mathbf{F i}$ \\
\hline$|28| 30 \cdot 13 \mid 66$ & & & & \begin{tabular}{|l|l|l|l|}
$30 \cdot 16$ & 67 & 69 & 59
\end{tabular} & & E. & P.M. N.E. fine \\
\hline $2930 \cdot 1066$ & 66 & 54 & 12 & \begin{tabular}{|l|l|l|l|}
$50 \cdot 04$ & 68 & 54 \\
\end{tabular} & & N. & \\
\hline & 67 & 50 & 11 & \begin{tabular}{|l|l|l|l|l|}
30.06 & 67 & 68 & 59 \\
\end{tabular} & .22 & w. & \\
\hline $31 \mid 30 \cdot 0$ & 166 & $|58|$ & 8 & $|30 \cdot 20 \quad 67,68| 60 \mid$ & & NE. & Fine, rain at $n$ \\
\hline
\end{tabular}

\begin{tabular}{llll}
\multicolumn{2}{r}{ Presnure. } & \multicolumn{2}{c}{ Corr $^{d}$ for Expan } \\
Max. $30 \cdot 24$ & at & $65^{\circ}$ & $=30 \cdot 155$ \\
Min. 29.74 & 66 & $=29 \cdot 655$ \\
Mean 30.063 & 66 & $=29.978$
\end{tabular}

Temperature

Temperature.
Max. $\ldots \ldots \ldots \ldots \ldots \ldots \ldots \ldots \ldots, 69^{\circ}$
Min. $\ldots \ldots \ldots \ldots \ldots \ldots \ldots \ldots, 55$

Mean $. . . \ldots \ldots \ldots \ldots \ldots \ldots \ldots \ldots, 63.2$
Max, $65^{\circ} \quad$ Min. $48^{\circ}$

Max. $17^{\text {Dryness. }}$ Min. 1

Rain 3.29 inches.

tVinds.

N. N.E. E. S. SW. W. NW.

$\begin{array}{lllllll}2 & 17 & 2 & 1 & 1 & 7 & 1\end{array}=31$.

Remarks.-The former part of the month unusually wet; the latter cold but seasonable. 
374 Dr. Heineken's Meteorological Register kept at Funchal

J U N E 1826.

\begin{tabular}{|c|c|c|c|c|c|c|c|c|c|}
\hline \multicolumn{4}{|c|}{10 o'Clock A.M. } & \multicolumn{3}{|c|}{10 o'Clock P.M. } & \multirow{3}{*}{ Rain. } & \multirow{3}{*}{ Wind } & \multirow{3}{*}{ Weather. } \\
\hline \multirow{2}{*}{$\begin{array}{l}\text { Barometer. } \\
\text { Bar. Th. }\end{array}$} & \multicolumn{3}{|c|}{ Hygrometer } & \multicolumn{2}{|c|}{ Barometer. } & \multirow{2}{*}{ Ther. } & & & \\
\hline & Air. & D.P. & Dry. & Bar. & Th. & & & & \\
\hline $1|30 \cdot 19| 67$ & 67 & 58 & $\overline{9}$ & $30 \cdot 17$ & 67 & 68 & & N. & Cloudy \\
\hline $2|30 \cdot 13| 66 \cdot 5$ & 67 & 55 & 12 & $30 \cdot 10$ & 67 & $68 \mid 61$ & & w. & Fin \\
\hline $3|30 \cdot 14| 67$ & 67 & 57 & 10 & $30 \cdot 22$ & 67 & $68 \mid 62$ & & w. & Small rain \\
\hline $4|30 \cdot 29| 67$ & & & & $30 \cdot 31$ & 68 & $69 \mid 59$ & 02 & E. & Fine \\
\hline $5|30 \cdot 29| 68$ & & & & $30 \cdot 27 \mid$ & 69 & $69 \mid 60$ & & NE. & Fine \\
\hline $6 \mid 30 \cdot 2268$ & 68 & 58 & 10 & $30 \cdot 21$ & 68 & $69|63|$ & & NE. & Overcast \\
\hline $7|30 \cdot 17| 68 \cdot 5$ & 68 & 59 & 9 & $30 \cdot 13$ & 69 & $68 \mid 62$ & & NE. & Overc \\
\hline $8|30 \cdot 07| 68$ & 68 & 57 & 11 & $30 \cdot 05$ & 69 & $69|59|$ & & w. & Overcast \\
\hline $9|50 \cdot 05| 68$ & 68 & 50 & 8 & $30 \cdot 06$ & 69 & $69 \mid 61$ & & N. & Ov \\
\hline $1030 \cdot 10 \mid 68 \cdot 5$ & 69 & 59 & 10 & $30 \cdot 19$ & 68 & $69|60|$ & & NE. & Ove \\
\hline \begin{tabular}{|l|l|l|}
11 & $30 \cdot 21$ & $68 \cdot 5$
\end{tabular} & 68 & 54. & 14 & $30 \cdot 30$ & $68 \cdot 5$ & $69 \mid 59$ & & E. & Overcast \\
\hline $12|30 \cdot 30| 68$ & 69 & 55 & 14 & $30 \cdot 28$ & 68 & $69|59|$ & & E. & Fine \\
\hline $3|30 \cdot 27| 68$ & 68 & 56 & 12 & $30 \cdot 24$ & 68 & 69961 & & NE. & Fine \\
\hline $40 \cdot 1768$ & $68 \mid$ & 60 & 8 & $30 \cdot 13$ & 68 & $6962 \mid$ & & N. & Fine \\
\hline $15 \mid 30 \cdot 1069$ & 69 & 62 & 7 & $30 \cdot 13$ & 70 & $7064 \mid$ & & W. & Thick \& overc. \\
\hline $16|30 \cdot 16| 69$ & 69 & 1 & 8 & $30 \cdot 18$ & 70 & 7064 & & w. & Thick \& overc. \\
\hline $17|30 \cdot 20| 69 \cdot 5$ & $69 \mid$ & 59 & 10 & $30 \cdot 16$ & 69 & $\mid \begin{array}{l}z 1|63| \\
\mid\end{array}$ & & w. & $\mathrm{Clo}$ \\
\hline $18|30 \cdot 12| 69 \cdot 5$ & $69 \mid$ & 57 & 12 & $30 \cdot 10$ & 69 & $7061 \mid$ & & w. & Clo \\
\hline $19 \mid 30 \cdot 0769$ & 69 & 63 & 6 & $30 \cdot 06$ & 69 & $|7165|$ & & w. & Cloudy \\
\hline $20|30.05| 70$ & 70 & 62 & 8 & $30 \cdot 09$ & 70 & $7163 \mid$ & & w. & Fine \\
\hline $130 \cdot 07 \mid 69 \cdot 5$ & 70 & 60 & 10 & $30 \cdot 10$ & 70 & $71|63|$ & & NW. & Fit \\
\hline $22 \mid 30^{\circ} \cdot 10 / 70$ & $70 \mid$ & 58 & 12 & $30 \cdot 10$ & 70 & $7064 \mid$ & & NW. & Overcast \\
\hline $23|30 \cdot 10| 70$ & & & & $30 \cdot 15$ & 70 & $71 \mid 62$ & & NW & Overcast \\
\hline $24|30 \cdot 15| 70$ & 70 & ol & 8 & $30 \cdot 17$ & 70 & $70 \mid 64$ & & NE. & Overcast \\
\hline $25|30 \cdot 15| 69 \cdot 5$ & 69 & 58 & 11 & $30 \cdot 18$ & 70 & $70|65|$ & & NE. & $\mathrm{Or}$ \\
\hline $2630^{\circ} 20 / 70$ & 71 & 55 & 16 & $30 \cdot 23$ & 70 & $70|61|$ & & $\mathrm{NE}$. & Overcast \\
\hline $27|30 \cdot 21| 70$ & $70 \mid$ & 60 & 10 & $30 \cdot 20$ & 70 & $7163 \mid$ & & NE. & Fine \\
\hline $28|30 \cdot 20| 70$ & 71 & 65 & 6 & $30 \cdot 20$ & 70 & $78|64|$ & & E. & Fine \\
\hline $990 \cdot 17$ & 71 & 65 & 6 & $30 \cdot 20$ & 71 & 7363 & & E. & Very \\
\hline $30|30 \cdot 20| 71$ & $71 \mid$ & & 41 & $\left.x 0^{0}=4\right]$ & 72 & $|72| 63 \mid$ & & E. & Very fine \\
\hline
\end{tabular}

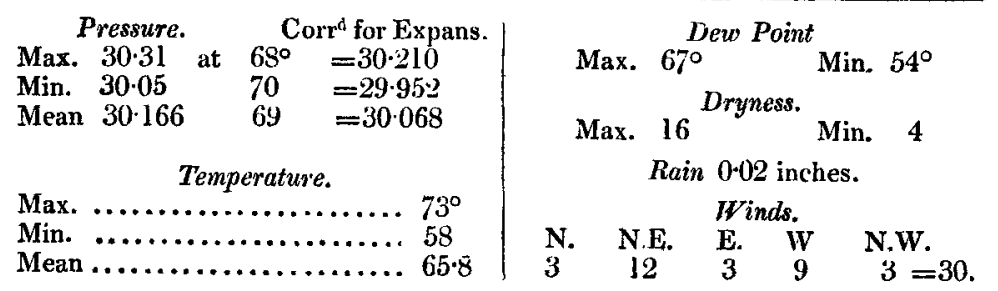

Remarks.-The former part of the month cloudy and cold; the latter warm and fine.

['To be continued.] 